\title{
OPTICAL PROPERTIES OF BULK GALLIUM OXIDE GROWN FROM THE MELT
}

\author{
M.G. Mynbaeva ${ }^{1,2}$, P.S. Shirshnev ${ }^{1}$, A.V. Kremleva ${ }^{1}$, A.N. Smirnov ${ }^{2}$, E.V. Ivanova ${ }^{2}$, \\ M.V. Zamoryanskaya², I.P. Nikitina², A.A. Lavrent'ev², K.D. Mynbaev ${ }^{1,2}$, \\ M.A. Odnoblyudov', D.A. Bauman', H. Lipsanen', V.I. Nikolaev'1,2, V.E. Bougrov' \\ and A.E. Romanov ${ }^{1,2}$ \\ ${ }^{1}$ ITMO University, Kronverkskiy 49, Saint-Petersburg 197101, Russia \\ ${ }^{2}$ Ioffe Institute, Polytechnicheskaya 26, Saint-Petersburg 194021, Russia
}

Received: May 03, 2018

\begin{abstract}
Results of the studies of the properties of single-crystalline bulk $\beta-\mathrm{Ga}_{2} \mathrm{O}_{3}$ grown from the melt are presented. High chemical purity and phase uniformity of the grown material are demonstrated. Raman spectroscopy studies confirmed low energies of optical phonons in $\beta-\mathrm{Ga}_{2} \mathrm{O}_{3}$, which makes it a promising material for laser optics applications.
\end{abstract}

\section{INTRODUCTION}

Wide-bandgap semiconductors are demanded in many applications, including fabrication of highly efficient solid-state lighting sources. Light-emitting diodes and laser diodes fabricated on the basis of wide-bandgap III-nitrides are already widely used in general lighting, full-colour display backlighting, and other industrial and consumer applications. Yet another wide-bandgap semiconductor, which currently attracts a strong interest, is monoclinic gallium oxide $\mathrm{Ga}_{2} \mathrm{O}_{3}$ with the bandgap value $\sim 4.9 \mathrm{eV}$ at $300 \mathrm{~K}$ [1-3]. Initially, $\beta-\mathrm{Ga}_{2} \mathrm{O}_{3}$ was considered as a material with great prospects in high-power electronics $[2,3]$. Indeed, gallium oxide looks like an ideal material for the fabrication of high-power diodes, as according to calculations it possesses electrical breakdown field of $8 \mathrm{MV} \mathrm{cm}^{-1}$, which is close to that of diamond $\left(10-20 \mathrm{MV} \mathrm{cm}^{-1}\right)[2,3]$. However, the results of recent research showed certain limitations in relation to carrier mobility in $\beta-\mathrm{Ga}_{2} \mathrm{O}_{3}$, which possibly may restrict its use in some electronic devices [4,5]. Still, gallium oxide possesses an im- portant advantage over other wide-bandgap semiconductor materials. This advantage is related to the possibility of manufacturing high-quality largesize bulk crystals using relatively inexpensive methods of growth from the melt. This surely allows for expanding application areas for the material. For example, currently the development of high-power ultra-fast lasers represents a strong interest. Such optoelectronic devices are demanded in broad-band communication systems, and of special interest are passive Q-switching lasers with saturable absorbers [6]. Currently these absorbers are mostly made of yttrium-aluminium garnet (YAG) doped with transition-metal ions. YAG, however, has relatively high maximum phonon energy, which hinders from achieving maximum laser efficiency because of losses associated with multi-phonon processes. Gallium oxide is considered to be a good replacement for YAG in laser optics applications [6], yet for the realization of all the advantages of $\beta-\mathrm{Ga}_{2} \mathrm{O}_{3}$ one needs to know the details of its properties in relation to a specific method of fabrication.

Corresponding author: M.G. Mynbaeva, e-mail:mgm@mail.ioffe.ru 
In this paper, we report on the results of the study of structural and optical properties of singlecrystalline bulk $\beta-\mathrm{Ga}_{2} \mathrm{O}_{3}$ grown from the melt. We have studied chemical and phase composition of the grown crystals, their phonon spectra and cathodoluminescence properties.

\section{EXPERIMENTAL DETAILS}

In contrast to widely-spread 'classic' $\mathrm{Ga}_{2} \mathrm{O}_{3}$ Czochralski growth, where $\mathrm{Ga}_{2} \mathrm{O}_{3}$ crystals are obtained via pulling the molten material out on a rotating seed [7-9], the seeding and the growth of our ingots proceeded directly in the crucible. Necessary conditions for the formation of the seed and for the following growth of the crystal were achieved via setting temperature gradients at every growth stage. This approach allows for commercial production of commercial-size sapphire crystals [10], suggesting that our technique may be eventually adapted for the growth of large gallium oxide crystals.

Characterization of the structure and composition of the material was performed with the use of $X$ ray diffractometry (XRD), Auger-electron spectroscopy (AES) and secondary-ion massspectroscopy (SIMS). Optical properties of the material were studied with the use of microcathodoluminescence (MCL) and Raman scattering spectroscopy. When preparing experimental samples, we used the ability of single-crystalline $\beta-\mathrm{Ga}_{2} \mathrm{O}_{3}$ to be easily cleaved along strong cleavage planes (100) or (001) under mechanical treatment $[11,12]$. This allowed us to fabricate samples in a form of platelets with given crystallographic orientation. Also, using mechanical cutting, we fabricated samples in a form of blocks (slabs) with faces corresponding to various crystallographic planes in $\beta-\mathrm{Ga}_{2} \mathrm{O}_{3}$ lattice. The dimensions of platelets obtained with cleaving were $\sim 0.2 \times 1 \times 7 \mathrm{~mm}$, those of the slabs, $\sim 3 \times 5 \times 10 \mathrm{~mm}$. To study the uniformity of the properties of the grown material, experimental samples were cut from various parts of the ingots.

\section{RESULTS AND DISCUSSION}

Analysis of chemical composition of the ingots was performed with the use of AES and SIMS. For AES, native oxides and possible surface contaminations were removed by bombarding the surface with electrons with $3 \mathrm{keV}$ energy. For studying the distribution of the chemical elements in the bulk of the sample (with the depth of the analysis down to 1200 $\mathrm{nm}$ ) we used sputtering of the surface with focused argon ion beam with $5 \mathrm{keV}$ ion energy. The results of the quantitative analysis of AES data, performed on the basis of the measured $\mathrm{Ga} / \mathrm{O}$ ratio, showed that the material under study was indeed stoichiometric $\mathrm{Ga}_{2} \mathrm{O}_{3}$. On the surface of the samples prepared with mechanic cutting and subsequent polishing, we detected carbon with concentration $<2.5$ at.\%. The low level of carbon contamination, which was in fact close to the detection limit of the used equipment (1 at.\%), indicated the readiness of the samples for further investigations.

Analysis of the distribution of residual impurities was performed on the basis of the SIMS data obtained with sputtering of the material with focused oxygen ion beam with $8 \mathrm{keV}$ ion energy. Concentrations of the impurities were calculated using coefficients of relative sensitivity. Commercially available high-purity $\beta-\mathrm{Ga}_{2} \mathrm{O}_{3}$ powder melt was used as a reference sample. No impurities with concentration exceeding detection limit of $1 \times 10^{13} \mathrm{~cm}^{-3}( \pm 5 \%)$ were found in the bulk of the studied samples.

Crystallographic orientation of crystal faces and structural quality of the samples were studied with the use of $X R D$. The XRD patterns were recorded in $(\omega)$ - and $(\omega, 2 \theta)-$ scanning modes. Dislocation-free $6 \mathrm{H}-\mathrm{SiC}$ (0001) single-crystal grown with the Lely method was used as a monochromator. Fig. 1 shows typical XRD patterns for samples obtained with cleaving along natural cleavage planes. As shown in Fig. 1a, the diffraction pattern obtained in $(\omega, 2 \theta)$ scan contains only peaks corresponding to reflections from (400) and (600) faces, which correspond to crystallographic planes in $\beta-\mathrm{Ga}_{2} \mathrm{O}_{3}$. The presence of two reflection peaks in $(\omega)$-scanning (Fig. 1b) is due to the imperfection of the cleaved surface of the sample, namely, to the presence of (100)-oriented steps. In this case, we observe diffraction both from the surface and from a region beneath it, as both these regions appear in the scanned area. The asymmetry of the peaks is indicative of the presence of micro-blocks with low mis-orientation in [100] direction. Low values of the full-widths at half-maximum (FWHM) of the recorded curves, which were limited to 40-50" as determined by the fitting of the experimental curves with Gaussians, were indicative of high perfection of the crystalline phase of the studied $\beta-\mathrm{Ga}_{2} \mathrm{O}_{3}$.

For MCL studies, a cathodoluminescence system combined with a scanning electron microscope was used. MCL spectra were recorded at the temperature $77 \mathrm{~K}$ with $10 \mathrm{keV}$ electron energy and 50 $\mathrm{nA}$ electron beam current. When collecting cathodoluminescence images, the diameter of the de-focused beam was $200 \mu \mathrm{m}$, when recording MCL 

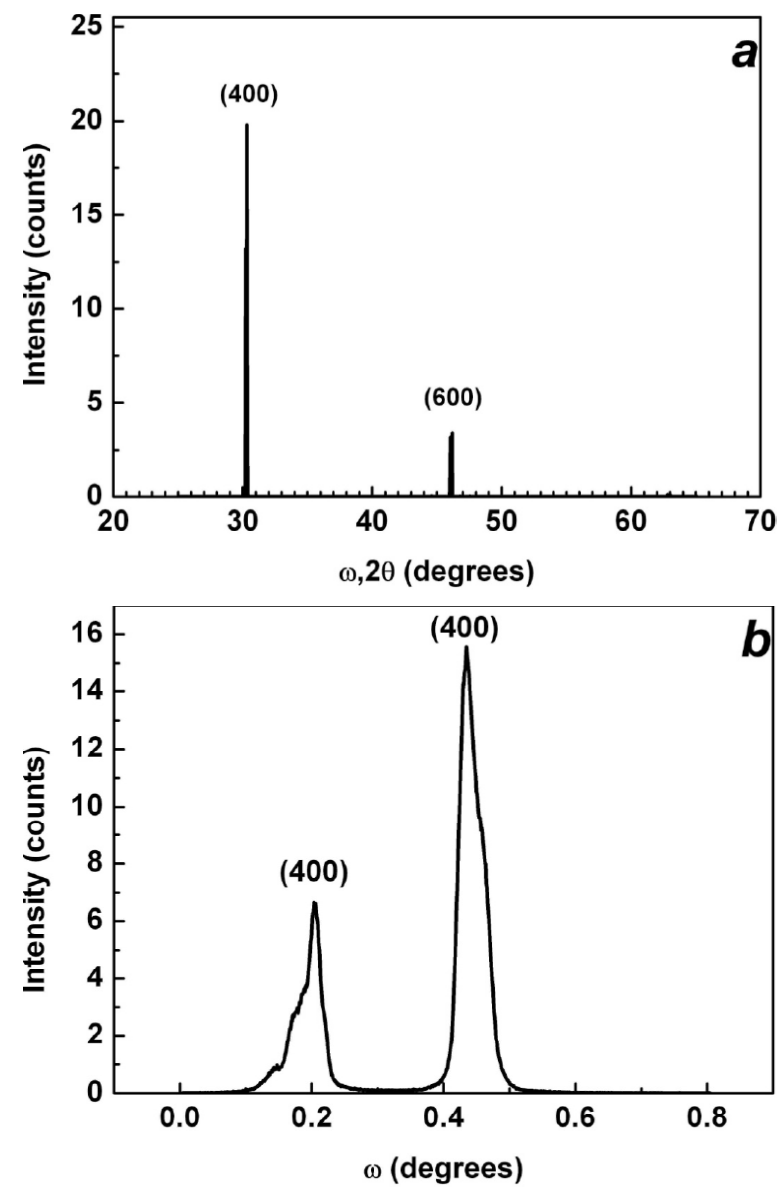

Fig. 1. XRD patterns of $\beta-\mathrm{Ga}_{2} \mathrm{O}_{3}$ obtained in $(\omega, 2 \theta)$ mode (a) and in $(\omega)$ mode $(b)$.

spectra, $2 \mathrm{~mm}$. All MCL spectra obtained from different sample faces (an example is shown in Fig. 2) exhibited wide (FWHM 600 to $700 \mathrm{meV}$ ) luminescence bands that could be fitted with the sum of two Gaussians peaked at $3.24 \mathrm{eV}$ (ultraviolet luminescence, UVL) and $2.85 \mathrm{eV}$ (blue luminescence, $\mathrm{BL})$, respectively. The UVL line dominated in all the spectra, yet in the spectra recorded from the face corresponding to (010) crystallographic plane, an increase in the relative intensity of the BL line was observed.

The obtained MCL spectra seem to be typical of monoclinic $\beta-\mathrm{Ga}_{2} \mathrm{O}_{3}[13-16]$. Indeed, emission spectrum of $\mathrm{Ga}_{2} \mathrm{O}_{3}$ typically does not contain near-bandedge luminescence line, but rather UVL and $\mathrm{BL}$ bands. The $\mathrm{BL}$ band is due to donor-to-acceptor optical transitions involving deep donors and acceptors (possible intrinsic donors in $\mathrm{Ga}_{2} \mathrm{O}_{3}$ are oxygen vacancies and gallium interstitials, while possible acceptors are gallium vacancies and their complexes with oxygen vacancies). Fig. 3 shows cathodoluminescence images of the samples. The image shown in Fig. 3a was obtained from (100) plane and shows uniform luminescence from all the

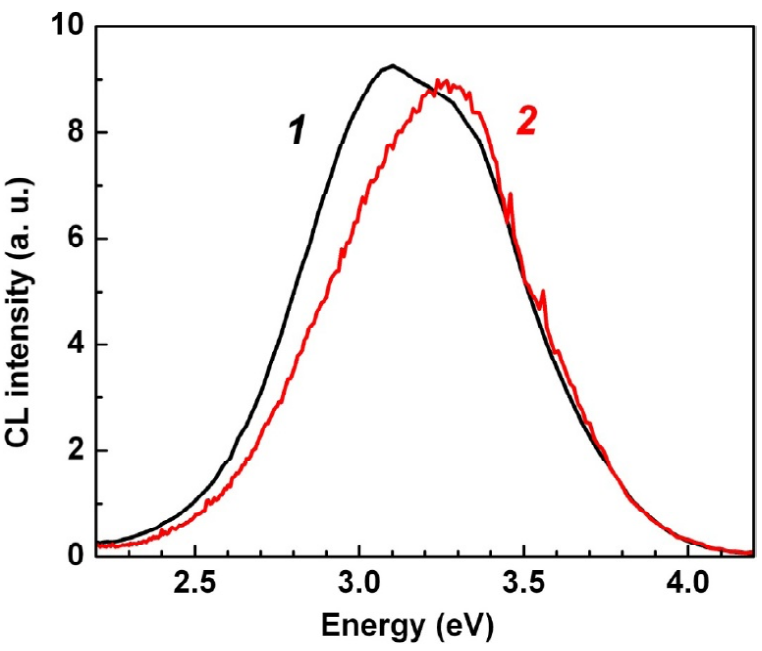

Fig. 2. Examples of $C L$ spectra registered from (010) plane (curve 1) and (100) plane (curve 2) of $\mathrm{Ga}_{2} \mathrm{O}_{3}$ samples.
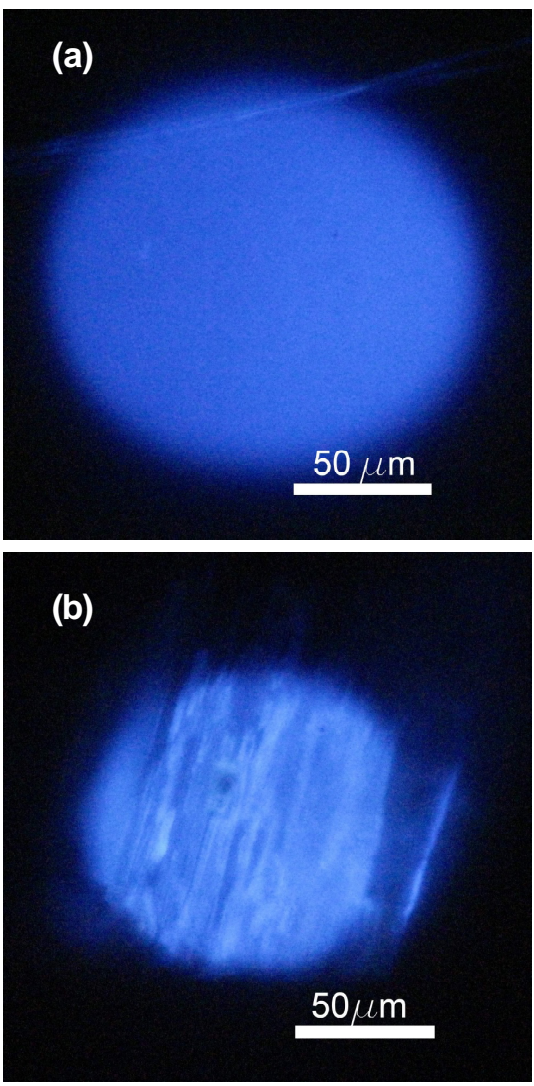

Fig. 3. Cathodoluminescence images of the surface of the studied $\beta-\mathrm{Ga}_{2} \mathrm{O}_{3}$ samples obtained from (100) (a) and (010) (b) planes.

area excited with the electron beam. The image shown in Fig. 3b was obtained from the (010) plane and demonstrates a strip-like emission pattern. This is possibly due to the presence of some extended defects decorated with intrinsic point defects.

The properties of the UVL band in $\mathrm{Ga}_{2} \mathrm{O}_{3}$ luminescence spectra usually do not depend on the type 


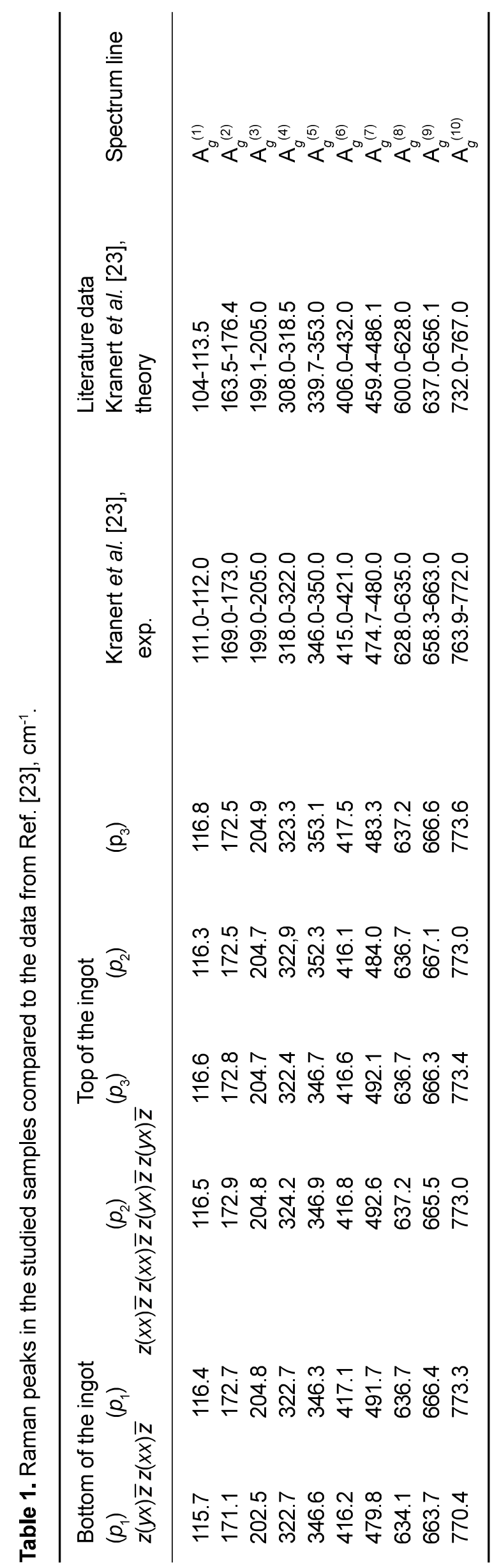


and concentration of impurities. The results of a number of experimental [13] and theoretical [17] investigations allowed for ascribing this band to recombination of free electrons and self-trapped holes (STH). Formation of STH is predicted for a wide range of oxides, including $\mathrm{Ga}_{2} \mathrm{O}_{3}$ [17], and recently was observed in $\mathrm{Ga}_{2} \mathrm{O}_{3}$ experimentally [18]. STH are formed when electron-phonon coupling becomes so strong that a hole in the valence band begins to introduce local distortion in the position of ions in the lattice. This distortion leads to the appearance of an electrostatic potential, which spatially localizes the hole in the valence band. It was established that formation of $\mathrm{STH}$, as a fundamental property of many oxide systems, defines low mobility of holes in these materials. In particular, some estimations showed that hole mobility in gallium oxide might not exceed $10^{-6} \mathrm{~cm}^{2} \mathrm{~V}^{-1} \mathrm{~s}^{-1}$ at $300 \mathrm{~K}$. Such low values of mobility obviously hinder manufacturing $p$ type gallium oxide with quality suitable for device fabrication [17-19].

Raman scattering studies were performed at the room temperature in backscattering geometry using T64000 Horiba Jobin Yvon Raman machine equipped with a confocal microscope. Raman spectra were excited (excitation wavelength $532 \mathrm{~nm}$ ) with a CW solid-state Nd:YAG laser with diode pumping. The laser beam was focused into a spot with $\sim 1 \mu \mathrm{m}$ in diameter; the power on the surface of the studied sample was $6 \mathrm{~mW}$. The Raman spectra were calibrated using spectral lines of a reference $\mathrm{Si}(111)$ sample.

$\beta-\mathrm{Ga}_{2} \mathrm{O}_{3}$ crystal is a sesquioxide belonging to the monoclinic family (space group: $\mathrm{C} 2 / \mathrm{m}$ ) [20]. Its crystal structure is base-centred monoclinic with the gallium ions occupying distorted octahedral and tetrahedral sites and the oxygen ions placed in a distorted cubic packing with three non-equivalent sites, with two threefold and one fourfold coordinated $\mathrm{O}$. According to polarization selection rules, in Raman spectra of $\mathrm{Ga}_{2} \mathrm{O}_{3}$ for $x x, y y, z z$, and $x y$ configurations only the $A_{g}$ optical modes are allowed [2123]. Raman modes typically observed in $\mathrm{Ga}_{2} \mathrm{O}_{3}$ can be divided into three groups: those due to high-frequency stretching and bending of $\mathrm{GaO}_{4}$ tetrahedra $\left(770-500 \mathrm{~cm}^{-1}\right)$, mid-frequency modes due to deformation of $\mathrm{Ga}_{2} \mathrm{O}_{6}$ octahedra $\left(480-310 \mathrm{~cm}^{-1}\right)$, and lowfrequency modes associated with libration and translation of tetrahedra-octahedra chains (below 200 $\mathrm{cm}^{-1}$ ). We studied a series of samples cut both from the top and from the bottom of the ingots. The measurements were performed in $z(x x) \bar{z}$ and $z(y x) \bar{z}$ scattering geometries at various points at (010) plane at a given polarization. In all the recorded spectra we observed similar sets of ten allowed $A_{g}$ modes. According to the data presented by Kranert et al. [23], this confirms that all the studied samples represented single-crystalline $\beta-\mathrm{Ga}_{2} \mathrm{O}_{3}$. As the $A_{g}$ lines for all the samples had similar spectral position, it was concluded that when being extracted from the melt, the material did not experience phase transformation and was phase-uniform throughout the whole ingot. The experimental data on the Raman modes acquired in this work are presented in Table 1 along with the data by Kranert et al. [23], both experimental and theoretical. Designations $(p 1) \ldots(p 3)$ in Table 1 relate to local spots analyzed on the surface of the sample. Fig. 4 shows, as an example, typical Raman scattering spectra obtained from the same spot of the sample in two polarization geometries. The spectra contain ten main peaks corresponding to optical-phonon scattering. The most intensive peak is located at $773.8 \mathrm{~cm}^{-1}$, this value is indeed much smaller than that in YAG (857 $\mathrm{cm}^{-1}$ ) [24]. The presented spectra show polarization dependence of peak intensity, such effect is ascribed to optical anisotropy (additionally affected by birefringence), which is typical of materials with low symmetry of crystal lattice, and of $\beta-\mathrm{Ga}_{2} \mathrm{O}_{3}$, in particular $[23,25]$.

Low energy of optical phonons surely may appear as a fundamental obstacle limiting electron mobility in gallium oxide $[4,5]$. It was believed that because effective mass in $\beta-\mathrm{Ga}_{2} \mathrm{O}_{3}$ is similar to that in $\mathrm{GaN}$, one might expect the $300 \mathrm{~K}$ electron mobility in these materials to be quite close (and it amounts to $1500 \mathrm{~cm}^{2} \mathrm{~V}^{-1} \mathrm{~s}^{-1}$ in $\mathrm{GaN}$ ). However, the maximum electron mobility in bulk single-crystalline $\beta-\mathrm{Ga}_{2} \mathrm{O}_{3}$ with the minimum density of dislocations, which was experimentally measured at $300 \mathrm{~K}$,

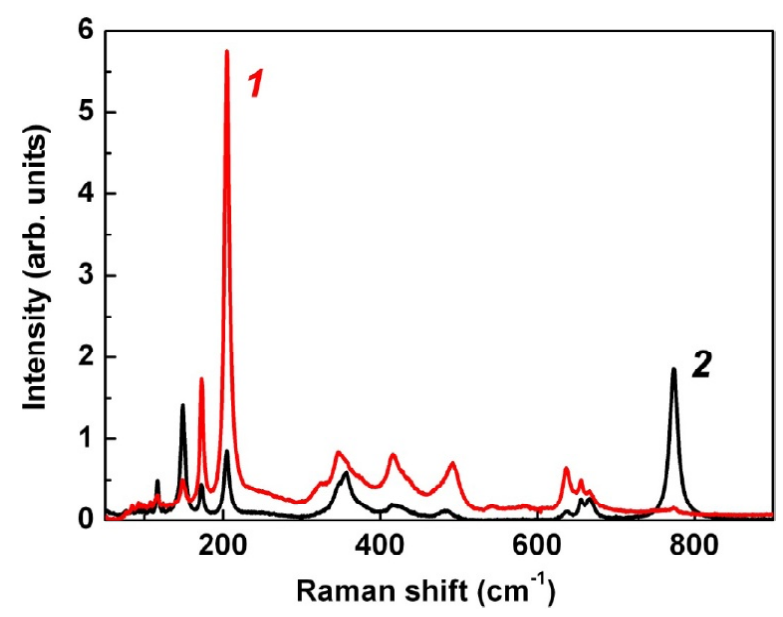

Fig. 4. Raman scattering spectra of $(010) \beta-\mathrm{Ga}_{2} \mathrm{O}_{3}$ sample obtained for $z(x x) \bar{z}$ (spectrum 1) and $z(y x) \bar{z}$ (spectrum 2) scattering geometries. 
appeared to be only $110-150 \mathrm{~cm}^{2} \mathrm{~V}^{-1} \mathrm{~s}^{-1}$, being ten times lower than that in $\mathrm{GaN}[4,5,26]$. The fundamental property of the material, which limits the mobility, is an extremely strong Fröhlich interaction, helped by the high ionicity of the chemical bonds and the low optical phonon energies [26]. This indeed limits ultimate parameters of prospective electronic devices based on $\beta-\mathrm{Ga}_{2} \mathrm{O}_{3}$. On the other hand, it is the low energy of optical phonons that makes $\beta-\mathrm{Ga}_{2} \mathrm{O}_{3}$ a promising material for laser optics applications, and the results presented in this work confirm that the used method of growth from the melt surely allows for manufacturing $\beta-\mathrm{Ga}_{2} \mathrm{O}_{3}$ with quality required in optical applications.

\section{CONCLUSION}

Results of characterization of bulk $\beta-\mathrm{Ga}_{2} \mathrm{O}_{3}$ crystals grown from the melt are presented. The studies of the properties of the material were performed with the use of X-ray Diffractometry, Auger Electron Spectroscopy, Secondary-lon Mass-Spectroscopy, Micro-Cathodoluminescence and Raman scattering spectroscopy. High chemical purity and phase uniformity of the grown $\beta-\mathrm{Ga}_{2} \mathrm{O}_{3}$ are demonstrated. Low energy of optical phonons was established with Raman scattering, and when combined with the results of the studies of structural properties, the obtained data confirm prospects of the studied material in laser optics technology.

\section{ACKNOWLEDGEMENTS}

This work was supported by the Ministry of Education and Science of the Russian Federation in the framework of the Federal Target Program "Research and development on priority directions of scientifictechnological complex of Russia for 2014-2020", the code 2017-14-582-0001, agreement No 14.581.21.0029 of October 23, 2017, unique ID RFMEFI58117X0029.

\section{REFERENCES}

[1] S.J. Pearton, J. Yang, P.H. Cary, F. Ren, J. Kim, M.J. Tadjer and M.A. Mastro // Appl. Phys. Rev. 5 (2018) 011301.

[2] M. Higashiwaki, K. Sasaki, H. Murakami, Y. Kumagai, A. Koukitu, A. Kuramata, T. Masui and S. Yamakoshi // Semicond. Sci. Technol. 31 (2016) 034001.

[3] S.I. Stepanov, V.I. Nikolaev, V.E. Bougrov and A.E. Romanov // Rev. Adv. Mater. Sci. 44 (2016) 63.
[4] N. Ma, N. Tanen, A. Verma, Z. Guo, T. Luo, H. Xing and D. Jena // Appl. Phys. Lett. 109 (2016) 212101.

[5] Y. Kang, K. Krishnaswamy, H. Peelaers and C.G. Van de Walle // J. Phys. Condens. Mat. 29 (2017) 234001.

[6] W. Mu, Y. Yin, Z. Jia, L.Wang, J. Sun, M. Wang, C. Tang, Q. Hu, Z. Gao, J. Zhang, N. Lin, S. Veronesi, Z. Wang, X. Zhao and X. Tao // RSC Adv. 7 (2017) 21815.

[7] Y. Tomm, P. Reiche, D. Klimm and T. Fukuda // J. Cryst. Growth 220 (2000) 510.

[8] Z. Gałązka, R. Uecker, K. Irmscher, M. Albrecht, D. Klimm, M. Pietsch, M. Brützam, R. Bertram, S. Ganschow and R. Fornari // Cryst. Res. Technol. 45 (2010) 1229.

[9] Z. Gałązka, K. Irmscher, R. Uecker, R. Bertram, M. Pietsch, A. Kwasniewski, M. Naumann, T. Schulz, R. Schewski, D. Klimm and M. Bickermann // J. Cryst. Growth 404 (2014) 184.

[10] M.I. Musatov // J. Opt. Technol. 76 (2009) 107.

[11] V.M. Bermudez // Chem. Phys. 323 (2006) 193.

[12] W. Mu, Z. Jia, Y. Yin, Q. Hu, J. Zhang, Q. Feng, Y. Hao and X. Tao // CrystEngComm 19 (2017) 5122.

[13] T. Harwig, F. Kellendonk and S. Slappendel // J. Phys. Chem. Solids 39 (1977) 675.

[14] L. Binet and J. Gourier // J. Phys. Chem. Solids 59 (1998) 1241.

[15] T. Matsumoto, M. Aoki, A. Kinoshita and T. Aono // Jap. J. Appl. Phys. 13 (1974) 1578.

[16] K. Shimamura, E.G. Villora, T. Ujiie and K. Aoki // Appl. Phys. Lett. 92 (2008) 201914.

[17] J.B. Barley, A. Janotti, C. Franchini and C.G. Van de Walle // Phys. Rev. B 85 (2012) 081109.

[18] B.E. Kananen, N.C. Giles, L.E. Halliburton, G.K. Foundos, K.B. Chang and K.T. Stevens // J. Appl. Phys. 122 (2017) 215703.

[19] A. Kyrtsos, M. Matsubara and E. Bellotti // Appl. Phys. Lett. 112 (2018) 032108.

[20] S. Geller // J. Chem. Phys. 33 (1960) 676.

[21] S. Geller // J. Sol. State Chem. 20 (1977) 209.

[22] D. Dohy and G. Lucazeau // J. Sol. State Chem. 45 (1982) 180. 
[23] C. Kranert, C. Sturm, R. Schmidt-Grund and M. Grundmann // Sci. Rep. 6 (2016) 35964.

[24] J.-J. Song, P.B. Klein, R.L. Wadsack, M. Selders, S. Mroczkowski and R.K. Chang // J. Opt. Soc. Amer. 63 (1973) 1135.
[25] M. Grundmann, C. Sturm, C. Kranert, S. Richter, R. Schmidt-Grund, C. Deparis and J. Zúñiga-Pérez // Phys. Stat. Sol. RRL 11 (2017) 1600295.

[26] A. Parisini, K. Ghosh, U. Singisetti and R. Fornari // Semicond. Sci. Technol. 33 (2018) 105008. 